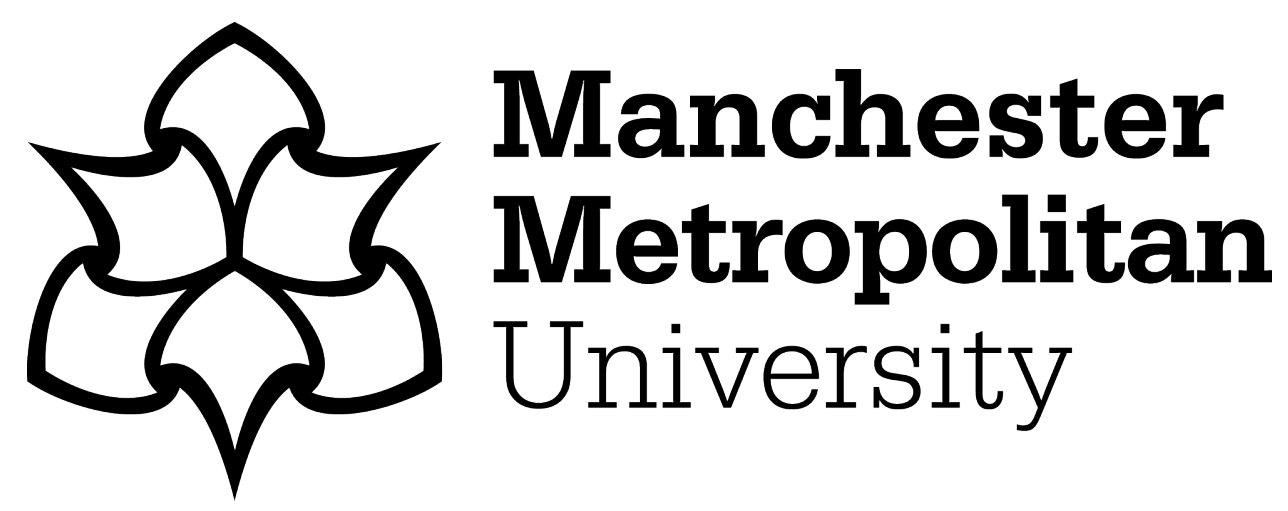

Zhang, X ORCID logoORCID: https://orcid.org/0000-0002-8790-0313, Wang, Z and Liu, Q (2018) Interpretation of Hot Spot Factor for Transformers in OD Cooling Modes. IEEE Transactions on Power Delivery, 33 (3). pp. 1071-1080. ISSN 0885-8977

Downloaded from: https://e-space.mmu.ac.uk/625851/

Version: Accepted Version

Publisher: IEEE

DOI: https://doi.org/10.1109/TPWRD.2017.2710087

Please cite the published version 


\section{Interpretation of Hot Spot Factor for Transformers in OD Cooling Modes}

Xiang Zhang, Zhongdong Wang, Member, IEEE, and Qiang Liu, Member, IEEE

\begin{abstract}
The hot-spot factor $(H)$ is a crucial component of the thermal diagram in IEC 60076-2 to derive the hot-spot temperature from the test data. In this paper, $H$ is expressed as the sum of two separable components, one relating to convection in the fluid domain and the other relating to conduction in the solid domain. Dimensional analyses on fluid flow and heat transfer show that both components are functions of dimensionless winding geometry, loss distribution, Re and Pr. The relationship between $H$ and $\operatorname{Re}$ and $P r$ for a fixed winding geometry with uniform loss distribution is obtained by conducting computational fluid dynamics (CFD) parametric sweeps and multilayer least-square based correlation. The correlation obtained is verified by the consistency between $H$ from new CFD simulations and those from the correlation. For nonuniform loss distribution, when the hot-spot is at the location of the highest power loss, $H$ is in a linear relationship with $Q$ and this linear relationship is coupled with a nonlinear relationship between $H$ and Re and Pr.
\end{abstract}

Index Terms - CFD, Dimensional analysis, disc winding, fluid flow, heat transfer, hot-spot factor, OD cooling mode, transformer.

\section{NOMENCLATURE}

$c_{p} \quad$ Oil specific heat at constant pressure $(\mathrm{J} /(\mathrm{kg} \cdot \mathrm{K}))$

$c_{p p} \quad$ Paper specific heat at constant pressure $(\mathrm{J} /(\mathrm{kg} \cdot \mathrm{K}))$

$c_{p c} \quad$ Copper specific heat at constant pressure $(\mathrm{J} /(\mathrm{kg} \cdot \mathrm{K}))$

$D_{h} \quad$ Hydraulic diameter at the pass inlet $\left(2 \times \mathrm{W}_{\text {duct }}\right)(\mathrm{m})$

$\Delta T \quad$ Temperature gradient across the paper insulation $(\mathrm{K})$

$\Delta x \quad$ The thickness of the paper insulation (m)

$g \quad$ Average temperature gradient $\left(\mathrm{T}_{\mathrm{aw}}-\left(\mathrm{T}_{\mathrm{to}}+\mathrm{T}_{\mathrm{bo}}\right) / 2\right)(\mathrm{K})$

$g^{\prime} \quad$ Local temperature gradient $\left(\mathrm{T}_{\text {bulk }}-\mathrm{T}_{\mathrm{to}}\right)(\mathrm{K})$

$h \quad$ Heat transfer coefficient at the hot-spot $\left(\mathrm{W} /\left(\mathrm{m}^{2} \cdot \mathrm{K}\right)\right)$

$h^{\prime} \quad$ Heat transfer coefficient of the winding $\left(\mathrm{W} /\left(\mathrm{m}^{2} \cdot \mathrm{K}\right)\right)$

$H \quad$ The hot-spot factor

$H_{d u} \quad$ The conductive component of $H$

$H_{v e} \quad$ The convective component of $H$

$k \quad$ Oil thermal conductivity $(\mathrm{W} /(\mathrm{m} \cdot \mathrm{K}))$

$k_{p} \quad$ Paper thermal conductivity $(\mathrm{W} /(\mathrm{m} \cdot \mathrm{K}))$

$k_{c} \quad$ Copper thermal conductivity $(\mathrm{W} /(\mathrm{m} \cdot \mathrm{K}))$

Manuscript received on $24^{\text {th }}$ November 2016, revised on $13^{\text {th }}$ February 2017 and $11^{\text {th }}$ April 2017, accepted on $25^{\text {th }}$ May 2017.

The authors are with the School of Electrical and Electronic Engineering at The University of Manchester, Manchester, M139PL, UK (e-mail: zhongdong.wang@ manchester.ac.uk).

\begin{tabular}{|c|c|}
\hline$N_{d e}$ & Number of domain elements \\
\hline$N u$ & The Nusselt number $\left(h \cdot \mathrm{D}_{\mathrm{h}} / k\right)$ \\
\hline$p$ & Static pressure $(\mathrm{Pa})$ \\
\hline $\operatorname{Pr}$ & The Prandtl number $\left(\mu \times c_{p} / k\right)$ \\
\hline$q^{\prime \prime}$ & Local heat flux at the hot-spot $\left(\mathrm{W} / \mathrm{m}^{2}\right)$ \\
\hline$q_{\max }^{\prime \prime}$ & Maximum local heat flux $\left(\mathrm{W} / \mathrm{m}^{2}\right)$ \\
\hline $\bar{q} "$ & Average heat flux on all winding discs $\left(\mathrm{W} / \mathrm{m}^{2}\right)$ \\
\hline$Q$ & Nonuniformity of power loss distribution $\left(q_{\max }^{\prime \prime} / \bar{q}^{\prime \prime}\right)$ \\
\hline$Q^{\prime}$ & Ratio of hot-spot heat flux to average flux ( $\left.q^{\prime \prime} / \bar{q}^{\prime \prime}\right)$ \\
\hline$r$ & Coordinate in radial direction \\
\hline $\mathrm{Re}$ & The Reynolds number $\left(u_{m} \times \rho \times D_{h} / \mu\right)$ \\
\hline$S$ & Nonuniformity of oil flow distribution \\
\hline$T$ & Temperature (K) \\
\hline$T_{a w}$ & Average winding temperature $(\mathrm{K})$ \\
\hline$T_{b o}$ & Bottom oil temperature $(\mathrm{K})$ \\
\hline$T_{b u l k}$ & Bulk oil temperature surrounding the hot-spot $(\mathrm{K})$ \\
\hline$T_{h s}$ & The hot-spot temperature on the copper $(\mathrm{K})$ \\
\hline$T_{h s}^{\prime}$ & The hot-spot temperature in the fluid domain $(\mathrm{K})$ \\
\hline$T_{t o}$ & Top oil temperature $(\mathrm{K})$ \\
\hline$u_{m}$ & Average oil velocity at winding pass inlet $(\mathrm{m} / \mathrm{s})$ \\
\hline$u_{r}$ & Radial velocity component (m/s) \\
\hline$u_{z}$ & Axial velocity component (m/s) \\
\hline$W_{d u c t}$ & Vertical duct width (m) \\
\hline$z$ & Coordinate in axial direction \\
\hline$\rho$ & Oil density $\left(\mathrm{kg} / \mathrm{m}^{3}\right)$ \\
\hline$\rho_{p}$ & Paper density $\left(\mathrm{kg} / \mathrm{m}^{3}\right)$ \\
\hline$\rho_{c}$ & Copper density $\left(\mathrm{kg} / \mathrm{m}^{3}\right)$ \\
\hline$\mu$ & Dynamic viscosity $(\mathrm{Pa} \cdot \mathrm{s})$ \\
\hline
\end{tabular}

\section{INTRODUCTION}

$\mathrm{T}$ HE hottest point in a transformer winding, usually referred to as the hot-spot, determines the loadability and lifetime of the transformer $[1,2]$. The hot-spot temperature could be measured directly using fiber-optic temperature sensors embedded in the windings during the manufacturing process [3-5]. It is shown that the hot-spot temperature may not be located in the topmost disc/turn, but in disc 2 or 3 from the topmost [4]. An accurate thermal modelling of transformers is therefore required to support the positioning of fiber-optic 
temperature sensors [5].

The fiber optic temperature sensors have not yet been widely installed for in-service transformers. The standard procedure for determining temperature distribution in a transformer is the temperature rise test, which, however, does not provide the hot-spot temperature directly. In the temperature rise test the top oil and bottom oil temperatures are measured either directly or indirectly, and the average winding temperature is derived from the winding resistance measurement. The hot-spot temperature can then be derived according to a thermal diagram, which has been used for decades $[1,6]$. In the thermal diagram, it is assumed that the oil temperature inside the winding increases linearly from the bottom to the top and there is a constant temperature gradient $(g)$ between the winding and the surrounding oil. The hot-spot is assumed to be located at the top of the winding. In order to compensate the error due to the linearity assumptions, the temperature gradient between the hot-spot and the top oil is made equal to $H \times g$, where $H$ is referred to as the hot-spot factor and is generally regarded as larger than unity. In so doing, the difficulty in determining the hot-spot temperature in transformer windings has been shifted to the determination of $H$. The inaccuracy in determining $H$ results in the uncertainty of the hot-spot temperature derived. In general, $H$ of 1.1 and 1.3 is used for distribution and transmission transformers when no specific information about the transformers is available.

$H$ is related to the oil flow distribution and power loss distribution in the winding. Therefore, $H$ is decomposed into factor $S$, describing the nonuniformity of the cooling, and factor $Q$, describing the nonuniformity of the power loss in IEC 60076-2:2011 [7]. However, how the $S$ and $Q$ factors should be quantified and how they should be formulated to characterize the variation of $H$ based on fundamental physical reasoning remains to be open questions.

A study of the decomposition of $H$ as the product of $S$ and $Q$ by using a detailed thermal-hydraulic network model showed that the $S$ factor and $Q$ factor as defined were interdependent [8]. Experimental determination of $H$ using fiber-optic temperature sensors for transformers of a wide range of power rating and cooling modes was conducted in [9]. It was found that $H$ obtained from 60 different load tests scattered from 0.51 to 2.06 [9]. A concept of effective hot-spot factor was raised in [10]. The effective hot-spot factor was reversely derived from a model that incorporates paper ageing mechanism and moisture accumulation effect into IEC thermal model [1]. It was found that the median of the effective hot-spot factors obtained from 35 scrapped power transformers was as high as 2.95 .

In this paper, analytical and numerical investigations of $H$ for transformers in OD cooling modes (pump-driven and directed oil flow) are presented. An interpretation of $H$ based on fundamental heat transfer and fluid flow analyses in the winding with the aid of dimensional analysis rather than the thermal diagram is provided in section II. Numerical investigation of $H$ using computational fluid dynamics (CFD) modelling is presented in section III, followed by discussion and conclusion in section IV and V, respectively.

\section{INTERPRETATION OF THE HOT-SPOT FACTOR}

\section{A. Winding Geometry under Investigation}

Disc-type transformer windings are investigated in this paper. Since the disc-type winding has an angular periodicity dictated by the number of radial spacers present along the circumference of the disc, the annular three-dimensional (3D) winding geometry can be reduced to the sector region between two sets of adjoining spacers. The fluid flow and heat transfer in the sector region is quasi two-dimensional (2D). Therefore, the winding geometry is further approximated into an axisymmetric $2 \mathrm{D}$ geometry in which the detailed phenomena in the vicinity of the spacers and strips are neglected. The differences in oil flow pattern and hot-spot temperature between 2D and 3D models can be alleviated by matching the governing dimensionless parameters, e.g. the ratio of the Grashof number to the Reynolds number square for oil natural (ON) cooling modes [11].

The investigated axisymmetric 2D winding model is shown in Fig. 1, where the detailed geometrical dimensions are presented. The winding model consists of 3 passes with 6 discs per pass and uniform horizontal duct height. The rounding radii of each strand are neglected to facilitate the meshing process, which will be detailed in section III part A.

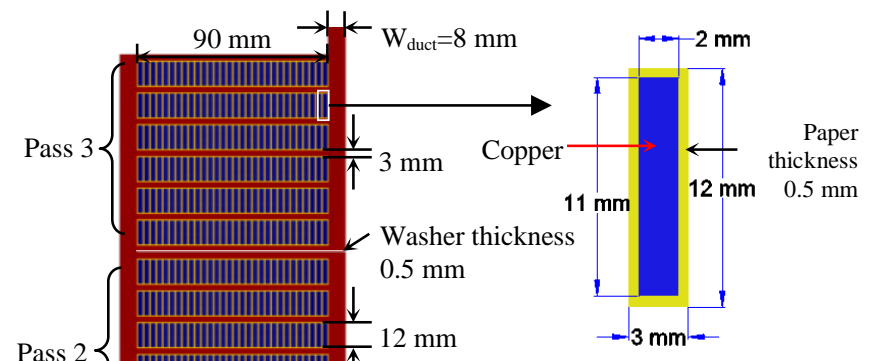

(b)

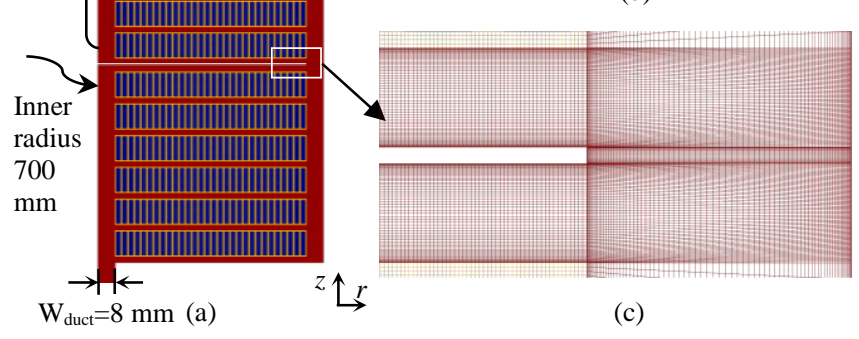

Fig. 1. Axisymmetric 2D winding model. (a) A three-pass winding model with 6 discs per pass. (b) Geometric details of the cross section of a strand. (c) Mesh details near a washer to be discussed in section III part A.

\section{B. Fluid Flow and Heat Transfer in the Winding}

Heat is generated in copper strands due to resistive losses and eddy current losses once the transformer is energized. The loss distribution in the winding can be obtained independently by considering the winding geometry, loading level and leakage flux distribution, etc. In this paper, we focus on the fluid flow and heat transfer processes in the winding with given loss distributions.

The thermal conduction within each copper strand is negligible due to the high thermal conductivity and the small size of the copper strand. The conduction through the paper insulation is governed by Fourier's law of conduction: 


$$
q^{\prime \prime}=\bar{q}^{\prime \prime} \cdot Q^{\prime}=k_{p} \frac{\Delta T}{\Delta x}
$$

where the $Q^{\prime}$ factor is the ratio of power loss at the hot-spot to the average power loss in the winding. The thermal convection in the fluid domain, which is the challenging part of the thermal analysis, is governed by the conservation laws of mass, momentum and energy.

The hot-spot temperature $\left(T_{h s}\right)$ in the winding can be regarded as the sum of the highest temperature in the fluid domain $\left(T_{h s}^{\prime}\right)$ and the temperature gradient across the paper insulation $(\Delta T)$.

$T_{h s}=T^{\prime}{ }_{h s}+\Delta T$

It is worth emphasizing that (2) is one way to express the hot-spot temperature, which will facilitate the following theoretical analyses. Equation (2) implicitly represents the fluid flow and heat transfer processes in the vicinity of the hot-spot because the determination of $T^{\prime}{ }_{h s}$ involves both the conduction in the solid domain and the convection in the fluid domain.

Based on (2), $H$ can be rewritten as:

$$
H=\frac{T_{h s}-T_{t o}}{T_{a w}-\left(\mathrm{T}_{t o}+\mathrm{T}_{b o}\right) / 2}=\frac{\Delta T}{g}+\frac{T_{h s}^{\prime}-T_{t o}}{g}=H_{d u}+H_{v e}
$$

The conductive component of $H, \Delta T / g$, is related to conduction in the paper insulation, referred to as $H_{d u}$. The convective component of $H,\left(T_{h s}^{\prime}-T_{t o}\right) / g$, is related to convection in the fluid domain, referred to as $H_{v e}$.

\section{1) Conductive Component of the Hot-Spot Factor}

Combining the conductive component, $\Delta T / g$, with (1) and noticing that the term $\bar{q} " / g$ can be interpreted as the overall heat transfer coefficient for the whole winding, $h^{\prime}$, we have:

$$
H_{d u}=\frac{\Delta T}{g}=\frac{\bar{q}^{\prime \prime} \cdot \Delta x}{g \cdot k_{p}} Q^{\prime}=\frac{h^{\prime} \cdot \Delta x}{k_{p}} Q^{\prime}
$$

Since $\Delta x$ can be linearly related to a characteristic length in the fluid domain and $k_{p}$ can also be linearly related to oil thermal conductivity, the term $h^{\prime} \cdot \Delta x / k_{p}$ is proportional to the Nusselt number associated with the overall heat transfer coefficient in the fluid domain. The Nusselt number for OD cooling modes is a function of the Reynolds number $(\mathrm{Re})$ and the Prandtl number (Pr) [12, 13]. Therefore, for a fixed winding geometry and a fixed power loss distribution in dimensionless senses, $H_{d u}$ would only be a function of $\operatorname{Re}$ and $\operatorname{Pr}[12,13]$.

\section{2) Convective Component of the Hot-Spot Factor}

The highest temperature in the fluid domain can be obtained according to Newton's law of cooling:

$$
q^{\prime \prime}=\bar{q}^{\prime \prime} \cdot Q^{\prime}=h\left(\mathrm{~T}_{h s}^{\prime}-\mathrm{T}_{b u l k}\right)
$$

where $T_{\text {bulk }}$ refers to oil bulk temperature surrounding the hot-spot and $h$ refers to the local heat transfer coefficient at the hot-spot in the fluid domain.

Combining the convective component, $\left(\mathrm{T}_{\mathrm{hs}}^{\prime}-\mathrm{T}_{\mathrm{to}}\right) / \mathrm{g}$, with (5), we have:

$H_{v e}=\frac{T_{h s}^{\prime}-T_{t o}}{g}=\frac{\bar{q}^{\prime \prime}}{g \cdot h} Q^{\prime}+\frac{T_{b u l k}-T_{t o}}{g}=\frac{h^{\prime}}{h} Q^{\prime}+\frac{g^{\prime}}{g}$

For a fixed winding geometry and a fixed power loss distribution in dimensionless senses, the ratio of the overall heat transfer coefficient, $h^{\prime}$, to the local heat transfer coefficient, $h$, and the ratio of the local oil temperature gradient, $g^{\prime}$, to the overall temperature gradient, $g$, are all functions of Re and Pr. This conclusion can be proved by conducting dimensional analyses on fluid flow and heat transfer in the fluid domain.

\section{Dimensional analyses on fluid flow and heat transfer \\ 1) Governing Equations in the Fluid Domain}

For OD cooling modes, the influence of buoyancy force is negligible as shown in [14]. Therefore, the conservation equations of mass, momentum and energy for steady, 2D flow of an imcompressible fluid with constant properties in cylindrical coordinates can be expressed by (7), (8), and (9), respectively [13].

$$
\begin{aligned}
& \frac{1}{r} \frac{\partial\left(\mathrm{ru}_{r}\right)}{\partial r}+\frac{\partial u_{z}}{\partial z}=0 \\
& \left\{\begin{array}{l}
\rho\left(\mathrm{u}_{r} \frac{\partial u_{r}}{\partial r}+\mathrm{u}_{z} \frac{\partial u_{r}}{\partial z}\right)=-\frac{\partial p}{\partial r}+\mu\left(\frac{\partial}{\partial r}\left(\frac{1}{r} \frac{\partial\left(\mathrm{ru}_{r}\right)}{\partial r}\right)+\frac{\partial^{2} u_{r}}{\partial z^{2}}\right) \\
\rho\left(\mathrm{u}_{r} \frac{\partial u_{z}}{\partial r}+\mathrm{u}_{z} \frac{\partial u_{z}}{\partial z}\right)=-\frac{\partial p}{\partial z}+\mu\left(\frac{1}{r} \frac{\partial}{\partial r}\left(\mathrm{r} \frac{\partial u_{z}}{\partial r}\right)+\frac{\partial^{2} u_{z}}{\partial z^{2}}\right)
\end{array}\right. \\
& u_{r} \frac{\partial T}{\partial r}+u_{z} \frac{\partial T}{\partial z}=\frac{k}{\rho c_{p}}\left(\frac{1}{r} \frac{\partial}{\partial r}\left(\mathrm{r} \frac{\partial T}{\partial r}\right)+\frac{\partial^{2} T}{\partial z^{2}}\right)
\end{aligned}
$$

Note that the viscous dissipation term for energy conservation is neglected because the oil velocity is small and therefore the viscous dissipation is negligible relative to advection and conduction.

\section{2) Nondimensionalization}

The dimensionless independent variables are defined as:

$r^{*}=\frac{r}{D_{h}} \quad$ and $\quad z^{*}=\frac{z}{D_{h}}$
$u_{r}^{*}=\frac{u_{r}}{u_{m}} \quad$ and $\quad u_{z}^{*}=\frac{u_{z}}{u_{m}}$
$p^{*}=\frac{p}{\rho u_{m}^{2}} \quad$ and $\quad T^{*}=\frac{T-T_{t o}}{T_{a w}-\left(\mathrm{T}_{t o}+\mathrm{T}_{b o}\right) / 2}=\frac{T-T_{t o}}{g}$

Substituting (10)-(12) into (7)-(9) and noticing that the hydraulic diameter $\left(D_{h}\right)$, the average oil velocity at the pass inlet $\left(u_{m}\right)$, and the lumped temperatures $\left(T_{a w}, T_{t o}, T_{b o}\right)$ are not functions of the coordinates, we can get the dimensionless forms of the governing equations as:

$$
\frac{1}{r^{*}} \frac{\partial\left(\mathrm{r}^{*} \mathrm{u}_{r}^{*}\right)}{\partial r^{*}}+\frac{\partial u_{z}^{*}}{\partial z^{*}}=0
$$




$$
\begin{aligned}
& \left\{\begin{array}{l}
\mathrm{u}_{r}^{*} \frac{\partial u_{r}^{*}}{\partial r^{*}}+\mathrm{u}_{z}^{*} \frac{\partial u_{r}^{*}}{\partial z^{*}}=-\frac{\partial p^{*}}{\partial r^{*}}+\frac{1}{\operatorname{Re}}\left(\frac{\partial}{\partial r^{*}}\left(\frac{1}{r^{*}} \frac{\partial\left(\mathrm{r}^{*} \mathrm{u}_{r}^{*}\right)}{\partial r^{*}}\right)+\frac{\partial^{2} u_{r}^{*}}{\partial z^{* 2}}\right) \\
\mathrm{u}_{r}^{*} \frac{\partial u_{z}^{*}}{\partial r^{*}}+\mathrm{u}_{z}^{*} \frac{\partial u_{z}^{*}}{\partial z^{*}}=-\frac{\partial p^{*}}{\partial z^{*}}+\frac{1}{\operatorname{Re}}\left(\frac{1}{r^{*}} \frac{\partial}{\partial r^{*}}\left(\mathrm{r}^{*} \frac{\partial u_{z}^{*}}{\partial r^{*}}\right)+\frac{\partial^{2} u_{z}^{*}}{\partial z^{* 2}}\right)
\end{array}\right. \\
& u_{r}^{*} \frac{\partial T^{*}}{\partial r^{*}}+u_{z}^{*} \frac{\partial T^{*}}{\partial z^{*}}=\frac{1}{\operatorname{Re} \cdot \operatorname{Pr}}\left(\frac{1}{r^{*}} \frac{\partial}{\partial r^{*}}\left(\mathrm{r}^{*} \frac{\partial T^{*}}{\partial r^{*}}\right)+\frac{\partial^{2} T^{*}}{\partial z^{*}}\right)
\end{aligned}
$$

\section{3) Flow and Temperature in the Fluid Domain}

For OD cooling modes, coolant flow determines heat transfer process, whereas the heat transfer has negligible influence on coolant flow distribution [14]. Therefore, fluid flow and heat transfer can be decoupled with fluid flow being determined first followed by the determination of the corresponding heat transfer.

From (13) and (14), it can be seen that for a fixed winding geometry, which is in a dimensionless sense with all the geometrical dimensions normalized against $\mathrm{D}_{\mathrm{h}}$, the dimensionless static pressure $\left(\mathrm{p}^{*}\right)$ and the dimensionless velocities $\left(\mathrm{u}_{\mathrm{r}}^{*}, \mathrm{u}_{\mathrm{z}}{ }^{*}\right)$ are functions of the dimensionless coordinates $\left(\mathrm{r}^{*}, \mathrm{z}^{*}\right)$ and the Reynolds number at the pass inlet. Here $\mathrm{Re}$ is the coefficient in the dimensionless flow differential equations. The static pressure drop coefficient over the winding model is related to $\mathrm{p}^{*}$ at the inlet and outlet of the winding and the oil flow proportion in a particular horizontal duct is related to $\mathrm{u}_{\mathrm{r}}{ }^{*}$ and $\mathrm{u}_{\mathrm{z}} *$ in that duct. Since the locations for pressure drop and duct flow proportion are fixed, the pressure drop coefficient flow proportion in a duct are only functions of Re at the winding pass inlet. It is detailed in [14] about the ways how pressure drop coefficient and flow distribution are controlled by $\mathrm{Re}$ and dimensionless geometrical parameters.

From (15), it can be seen that for a fixed winding geometry and a fixed power loss distribution in dimensionless senses, the dimensionless temperature $\left(T^{*}\right)$ is a function of the dimensionless coordinates $\left(\mathrm{r}^{*}, \mathrm{z}^{*}\right), \quad \mathrm{Re}$ and Pr. The dimensionless temperature at the hot-spot in the fluid domain, of which there can be only one location, is actually the convective component of the hot-spot factor $\left(H_{v e}\right)$. Therefore, $H_{v e}$ is only a function of Re and Pr.

\section{Determination of the Hot-Spot Factor}

From the aforementioned dimensional analyses, it can be concluded that for a fixed winding geometry and a fixed loss distribution in dimensionless senses both the conductive and convective components of $H$ are functions of Re and Pr. The power loss distribution in the winding or the $Q^{\prime}$ factor will affect $H$ directly. The magnitude of the power loss will affect $H$ only through its influences on Re and Pr. Combining (3), (4) and (6), we have:

$H=\left(\frac{h^{\prime}}{h}+\frac{h^{\prime} \cdot \Delta x}{k_{p}}\right) \cdot Q^{\prime}+\frac{g^{\prime}}{g}$

The ratios of $h^{\prime} / h, h^{\prime} \cdot \Delta x / k_{p}$ and $g^{\prime} / g$, are all functions of Re and $\mathrm{Pr}$, following similar analyses presented in section II part C. It can be seen from (16), $H$ is in a linear relationship with $Q^{\prime}$ and this linear relationship is coupled with the nonlinear relationship between $H$ and $\operatorname{Re}$ and $\operatorname{Pr}$. The quantitative relationship between $H$ and $Q^{\prime}$, Re and $\operatorname{Pr}$ can be determined by either experimentation or theoretical calculations.

\section{THEORETICAL DETERMinATION OF HOT SPOT FACTOR}

In this paper, theoretical approach based on CFD simulations and a subsequent correlation exercise is adopted to quantify the relationship between $H$ and $Q^{\prime}$, Re and Pr.

\section{A. CFD Simulations}

CFD models are established using commercial software COMSOL Multiphysics 5.2.

\section{1) Numerical models}

In the CFD models, conduction heat transfer is modeled in the solid domain (copper and paper), with copper and paper properties treated as temperature independent in the investigated temperature range $[11,16]$ :

$k_{c}=401(\mathrm{~W} /(\mathrm{m} \cdot \mathrm{K}))$

$k_{p}=0.19(\mathrm{~W} /(\mathrm{m} \cdot \mathrm{K}))$

$\rho_{c}=8933\left(\mathrm{~kg} / \mathrm{m}^{3}\right)$

$\rho_{p}=930\left(\mathrm{~kg} / \mathrm{m}^{3}\right)$

$\mathrm{c}_{p c}=385(\mathrm{~J} /(\mathrm{kg} \cdot \mathrm{K}))$

$\mathrm{c}_{p p}=1340(\mathrm{~J} /(\mathrm{kg} \cdot \mathrm{K}))$

For the convection heat transfer in the fluid domain, since the Reynolds numbers for practical winding cooling situation are smaller than the transition criterion to turbulence, no turbulence model is included in the CFD models. The continuity equation, Navier-stokes equations and the energy equation are solved directly, taking into account buoyancy force and without adopting Boussinesq approximation. It is worth mentioning that the use of Boussinesq approximation to take into account buoyancy forces brings negligible differences in flow distribution and temperature distribution as compared to a conjugate heat transfer model [16]. These equations solved in the CFD models differ from (7)-(9) in the way that the properties of the fluid, a mineral oil, are treated as functions of temperature. The oil properties are obtained from least-square curve fittings of the data provided by the oil manufacturer, given as follows:

$\rho=-0.6568 \times \mathrm{T}+1064$

$\mu=7.863 \times 10^{-5} \times \exp (632.0 /(\mathrm{T}-176.0))$

$k=-7.837 \times 10^{-5} \times T+0.1557$

$c_{p}=3.950 \times T+560.2$

where the temperatures are in Kelvin.

In this paper, conjugate heat transfer modeling as specified in [16] is adopted for the numerical investigation.

\section{2) Mesh Refinement Study}

In the CFD models, the meshes consist of rectangular layers, as illustrated in Fig. 1 (c). The rounding radii of the strands are omitted because the practical range of the rounding radii of the strands have negligible influences on oil flow distribution in the winding pass [14], whereas omitting the rounding radii can facilitate mesh control significantly. In the meshes, 4 elements 
are present to discretize the $0.5 \mathrm{~mm}$ paper thickness, as recommended in [11], and 256 elements are present to discretize each copper strand. In the fluid domain, the rectangular layers are structured in such a way that more elements are present close to the duct wall to capture the thermal and hydraulic boundary layers. Mesh refinement study of the fluid domain for the case of Re being 200 and Pr being 150 is performed. The tracking parameter for each mesh size or number of fluid domain elements $\left(N_{d e}\right)$ is $H$. The mesh refinement study results are shown in Table I.

TABLE I

MESh REFINEMENT STUDY RESULTS

\begin{tabular}{c|cccc}
\hline \hline Strategy & mesh 1 & mesh 2 & mesh 3 & mesh 4 \\
\hline$N_{d e}$ & 389,480 & 596,800 & 766,320 & $1,087,040$ \\
\hline$H$ & 1.1924 & 1.1869 & 1.1867 & 1.1869 \\
\hline $\begin{array}{c}\text { Computational } \\
\text { time }\end{array}$ & $\begin{array}{c}18 \\
\text { minutes }\end{array}$ & $\begin{array}{c}36 \\
\text { minutes }\end{array}$ & $\begin{array}{c}50 \\
\text { minutes }\end{array}$ & $\begin{array}{c}68 \\
\text { minutes }\end{array}$ \\
\hline \hline
\end{tabular}

As can be seen from Table I, the differences in $H$ obtained from the four mesh strategies are negligible. Nevertheless, a finer mesh size can better capture the hot-streak dynamics. Therefore, the mesh strategy corresponding to mesh 4 , which discretizes the $3 \mathrm{~mm}$ horizontal duct height with 60 elements and the $8 \mathrm{~mm}$ vertical duct width with 80 elements, is adopted for all the CFD models.

\section{B. Uniform Power Loss Distribution Cases}

Power losses in the winding conductor consist of resistive losses and eddy current losses. For a fixed winding geometry, the resistive losses are dictated by the current density and copper temperature and the eddy current losses are dictated by the leakage magnetic flux distribution and copper temperature. For power transformers, the current density in the copper wire ranges from 2-4 $\mathrm{A} / \mathrm{mm}^{2}$ [15], and the average winding temperature rise over ambient temperature is required to be not higher than $65 \mathrm{~K}$. For uniform-loss cases that account only the resistive losses, volumetric heat source of $3.397 \times 10^{5} \mathrm{~W} / \mathrm{m}^{3}$, corresponding to the condition of current density being 4 $\mathrm{A} / \mathrm{mm}^{2}$ and copper resistance at $85^{\circ} \mathrm{C}$, is prescribed to each copper strand.

\section{1) Parametric Sweeps and Correlations}

For the fixed winding geometry shown in Fig. 1 with a uniform loss distribution $\left(Q=Q^{\prime}=1\right)$, the hot-spot factor is a function of $\mathrm{Re}$ and $\mathrm{Pr}$, which are defined at the average temperature of the top oil and bottom oil temperatures. The bottom oil temperature is a given condition and the top oil temperature can be calculated according to energy conservation. The quantitative relationship between $H$ and $\mathrm{Re}$ and Pr can be obtained by conducting CFD parametric sweeps and correlating the $H$ results from CFD with Re and Pr.

\section{a. Parametric sweeps}

For OD cooling modes, Re is set to range from 200 to 1200. For the mineral oil whose properties as functions of temperature are shown in (17)-(20), when average oil temperature increases from $30^{\circ} \mathrm{C}$ to $80^{\circ} \mathrm{C}$, Pr decreases from 151 to 43. Therefore, in the parametric sweeps, Pr ranges from 60 to 150 . The discrete $\operatorname{Re}$ and Pr swept are shown in Table II.
TABLE II

RANGES OF THE SWEPT DIMENSIONLESS PARAMETERS

\begin{tabular}{c|l}
\hline \hline $\operatorname{Re}$ & $200,300,400,500,600,700,800,900,1000,1100,1200$ \\
\hline $\operatorname{Pr}$ & $60,100,150$ \\
\hline \hline
\end{tabular}

All the combinations of Re and Pr in Table II, in total 33 cases, are simulated to quantify the relationship between $H$ and $\mathrm{Re}$ and Pr. It is worth emphasizing that according to the principle of dimensional analysis it is Re and Pr themselves rather than their components that determines $H$ [14].

It is concluded in [14] that with the increase of Re, oil flow distribution in the winding pass gets less uniform with more oil flowing through the top horizontal ducts in a pass and hence bottom ducts suffering from oil starvation. Therefore, the temperature distribution could become less uniform with the increase of Re. Fig. 2 shows an example, where Pr of both cases is fixed to be 100 . When $\mathrm{Re}$ is 200 , temperature distribution in the winding is rather uniform as a result of rather uniform flow distribution. For the case of Re being 1200, temperature distribution gets distorted because oil starvation in the bottom ducts of each pass causes localized overheating. The flow distributions in the top pass for the two cases are shown in Fig. 3. Furthermore, reverse flow at the bottom of the pass can occur with a further increase of $\operatorname{Re}[14]$.

The variations of $H$ with Re and Pr from the CFD parametric sweeps are shown in Fig. 4. Because the loss distribution is uniform, the hot-spot temperature is determined by oil flow distribution. As can be seen in Fig. 4, when $\operatorname{Re}<600, H$ fluctuates slightly as the hot-spot temperature shifts from the middle to the bottom of pass 3 with the increase of Re. When $\mathrm{Re}>600, H$ increases with increasing Re due to more distorted flow distribution. In addition, $\mathrm{Pr}$ in its practical range is much less influential than Re.

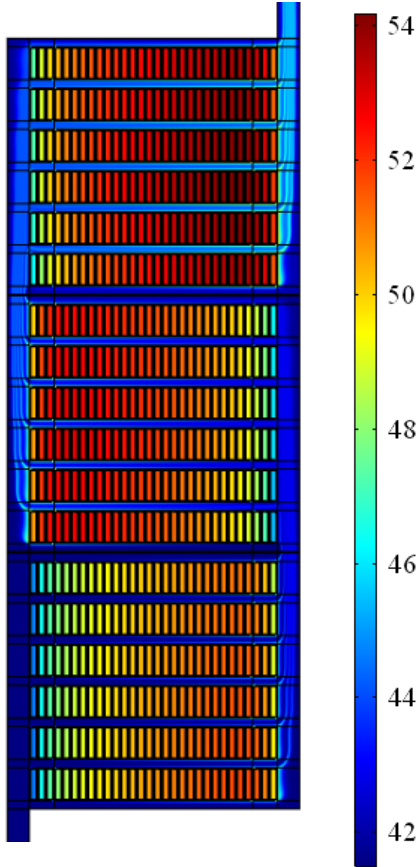

(a)

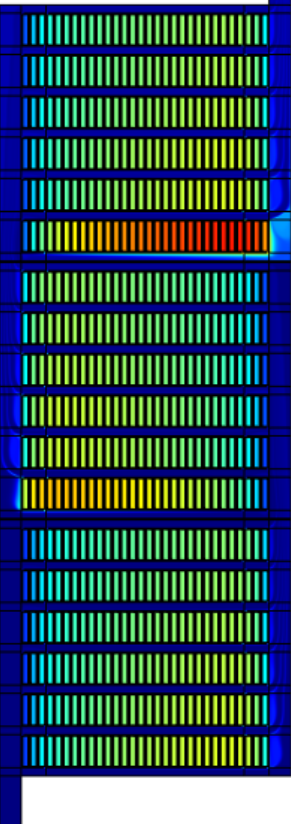

(b)
Fig. 2. Temperature distribution in the winding for $\operatorname{Pr}=100$ in Celsius. (a) $\mathrm{Re}=200$; (b) $\mathrm{Re}=1200$. (a) and (b) share the same colorbar. 

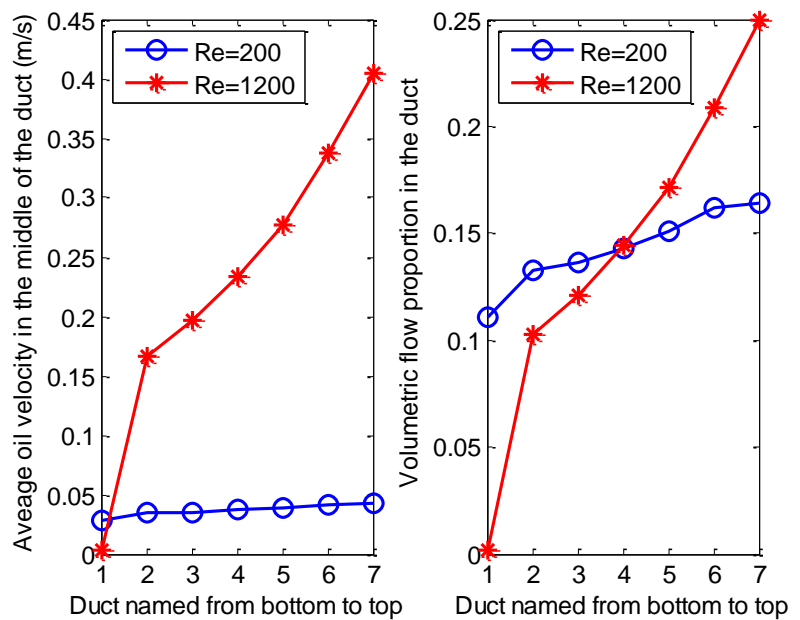

Fig. 3. Flow distribution in the winding model.

\section{b. Correlations}

The hot-spot factors obtained from the CFD parametric sweeps are correlated with Re and Pr by multilayer least-square curve fittings in the form of:

$$
\left\{\begin{array}{l}
H=\exp \left(a_{1}\left(\frac{\mathrm{Re}}{1000}\right)^{3}+a_{2}\left(\frac{\mathrm{Re}}{1000}\right)^{2}+a_{3}\left(\frac{\mathrm{Re}}{1000}\right)+a_{4}\right) \\
a_{i}=b_{i 1}\left(\frac{\operatorname{Pr}}{100}\right)^{2}+b_{i 2}\left(\frac{\operatorname{Pr}}{100}\right)+b_{i 3} \quad i=\{1,2,3,4\}
\end{array}\right.
$$

where the correlation coefficients $\mathrm{a}_{1}$ to $\mathrm{a}_{4}$ are dummy coefficients, which are dictated by $\operatorname{Pr}$ and the corresponding $b$ coefficients. With all the $b$ coefficients provided in Table III, $H$ for any combination of Re and $\operatorname{Pr}$ can be obtained from (21). With the least-square curve fitting strategy, the maximum relative error between $H$ obtained from (21) and that from the CFD simulations is $1.34 \%$. It is worth mentioning the correlation coefficients in Table III are specific to the winding shown in Fig. 1 because the geometry of the winding defines boundary conditions for (13)-(15) and therefore results in different solutions.

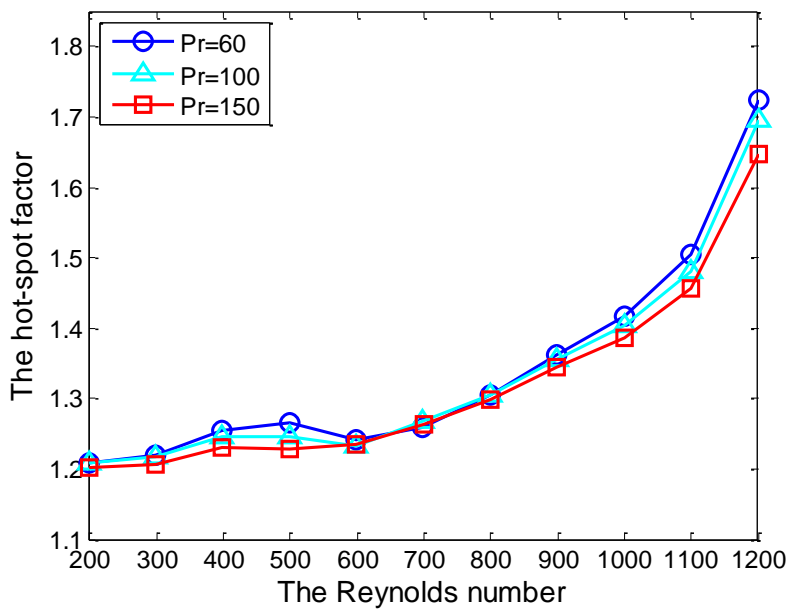

Fig. 4. Variation of $H$ with Re and $\operatorname{Pr}$.
TABLE III

CORRELATION COEFFICIENTS

\begin{tabular}{c|ccc}
\hline \hline$b_{i j}$ & $j=1$ & $j=2$ & $j=3$ \\
\hline$i=1$ & 0.0217 & -0.3199 & 1.0633 \\
$i=2$ & -0.0898 & 0.6795 & -1.7344 \\
$i=3$ & 0.0931 & -0.4571 & 0.9839 \\
$i=4$ & -0.0300 & 0.0896 & 0.0408 \\
\hline \hline
\end{tabular}

\section{2) Verifications and Predictions}

In order to verify the correlation (21) obtained, new CFD simulations are performed for comparison purposes. For the new simulations, winding pass inlet oil temperature is fixed to be $40^{\circ} \mathrm{C}$ and pass inlet average oil velocity is set to range from $0.2 \mathrm{~m} / \mathrm{s}$ to $0.5 \mathrm{~m} / \mathrm{s}$ with an interval of $0.1 \mathrm{~m} / \mathrm{s}$. Two uniform loss distributions are tested. One is of volumetric power density $3.397 \times 10^{5} \mathrm{~W} / \mathrm{m}^{3}$ corresponding to the current density of 4 $\mathrm{A} / \mathrm{mm}^{2}$ with copper resistance at $85{ }^{\circ} \mathrm{C}$. The other is of volumetric power density $7.643 \times 10^{5} \mathrm{~W} / \mathrm{m}^{3}$ corresponding to the current density of $6 \mathrm{~A} / \mathrm{mm}^{2}$ with copper resistance at $85^{\circ} \mathrm{C}$. In all these CFD cases, the Prandtl numbers are approximately $100 \pm 7$.

The comparisons of $H$ obtained from the new CFD simulations and the prediction curve obtained from correlation (21) with Pr being 100 are shown in Fig. 5 (a). It can be seen that $H$ from the CFD simulations with different power losses fall close to the prediction curve with maximum absolute error being 0.018 and maximum relative error being $1.39 \%$. It is worth mentioning that this maximum relative error of $1.39 \%$ is close to the maximum relative error of $1.34 \%$ when deriving the correlation (21) and this further verifies the applicability of the correlation and that $H$ is determined by the loss distribution pattern rather than the magnitude of the losses.

The hot-spot temperature can be calculated from the definition of $H$. In the calculations, the top oil and average oil temperatures are derived from energy conservation and the average winding temperature is taken from the CFD simulations, which is permissible because in practice average winding temperature can be derived from winding resistance measurements. The comparisons of $T_{h s}$ derived from the correlation of $H$ and those from the new CFD simulations are shown in Fig. 5 (b). It can be seen that $T_{h s}$ from both methods are almost identical for both power losses with maximum deviation being $0.2^{\circ} \mathrm{C}$.

The hot-spot temperature shown in Fig. 5 (b) decreases at first with the increase of $\mathrm{Re}$, which is proportional to total oil flow rate for a fixed winding geometry and a fixed oil type, then the hot-spot temperature starts to level off. In fact, with a further increase of Re, the hot-spot temperature tends to increase. This varying trend of $T_{h s}$ with Re is caused by the variation of oil flow distribution in the winding pass. With a higher Re, i.e. a higher flow rate, the flow distribution gets more distorted and causes localized overheating and higher $H$. This influence of the flow distribution on $T_{h s}$ is characterized by the variation of $H$ illustrated in Fig. 4 and more specifically in Fig. 5 (a). 


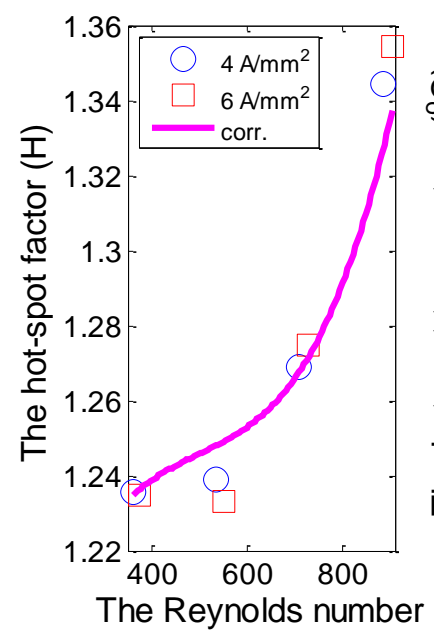

(a)

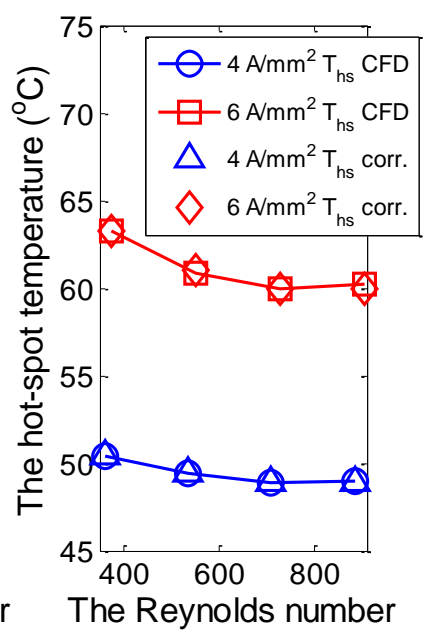

(b)
Fig. 5. Comparisons of $H$ and the hot-spot temperatures obtained from theoretical calculations and new CFD simulations.

\section{Nonuniform Power Loss Distribution Cases}

In a transformer winding, the eddy current losses concentrate on the top and bottom of the winding. For the 3-pass winding model shown in Fig. 1, extra losses are prescribed to the top three discs to represent the presence of eddy current losses. The 3 -pass winding model can therefore be regarded as the top 3 passes of a practical winding. All the discs except for the top three maintain the resistive loss of $3.397 \times 10^{5} \mathrm{~W} / \mathrm{m}^{3}$. The losses in the top three discs from the top of the winding are raised to the power ratios of $\mathrm{n}_{1}, \mathrm{n}_{2}, \mathrm{n}_{3}$ relative to the resistive loss. Loss variation along the radial direction is ignored. Five sets of nonuniform loss cases shown in Fig. 6 are simulated by CFD simulations. The corresponding $Q$ values are shown in Table IV

Since $\mathrm{Pr}$ is much less influential than $\mathrm{Re}, \mathrm{Pr}$ is controlled to be 100 and Re ranges from 200 to 1000 , which is a practical range of $\mathrm{Re}$, with an interval of 200 in these simulations. The hot-spot temperatures of these cases locate at the top disc because of the highest extra losses at that disc. Therefore, $Q$ equals $Q^{\prime}$ and $H$ is a function of $\operatorname{Re}$ and $Q$. The variations of $H$ and $T_{h s}$ with $\operatorname{Re}$ and $Q$ for these cases are shown in Fig. 7 (a) and (b), respectively. It can be seen that both $H$ and $T_{h s}$ decrease monotonically with the increase of $\mathrm{Re}$, and increase monotonically with the increase of $Q$ factor.

TABLE IV NONUNIFORM-LOSS CASES

\begin{tabular}{c|ccccc}
\hline \hline & Case 1 & Case 2 & Case 3 & Case 4 & Case 5 \\
\hline$n_{1}$ & 1.3 & 1.6 & 1.8 & 2.0 & 2.2 \\
$n_{2}$ & 1.2 & 1.4 & 1.5 & 1.5 & 1.8 \\
$n_{3}$ & 1.1 & 1.2 & 1.2 & 1.2 & 1.2 \\
$Q$ & 1.27 & 1.54 & 1.71 & 1.89 & 2.05 \\
\hline \hline
\end{tabular}

$\mathrm{n}_{1}$ refers to the ratio of power loss at the top disc to the average power loss, $\mathrm{n}_{2}$ refers to the ratio for the second disc from the top, $\mathrm{n}_{3}$ refers to the ratio for the third disc from the top.

The variations of $H$ with $Q$ for different Reynolds numbers are shown in Fig. 8. As can be seen, $H$ increases linearly with increasing $Q\left(Q^{\prime}\right)$, verifying the linear relationship predicted in (16). In addition, the slops for different Reynolds numbers are almost identical, indicating that the term $h^{\prime} / h+h^{\prime} \cdot d x / k_{p}$ is quite constant for the five Reynolds numbers investigated.

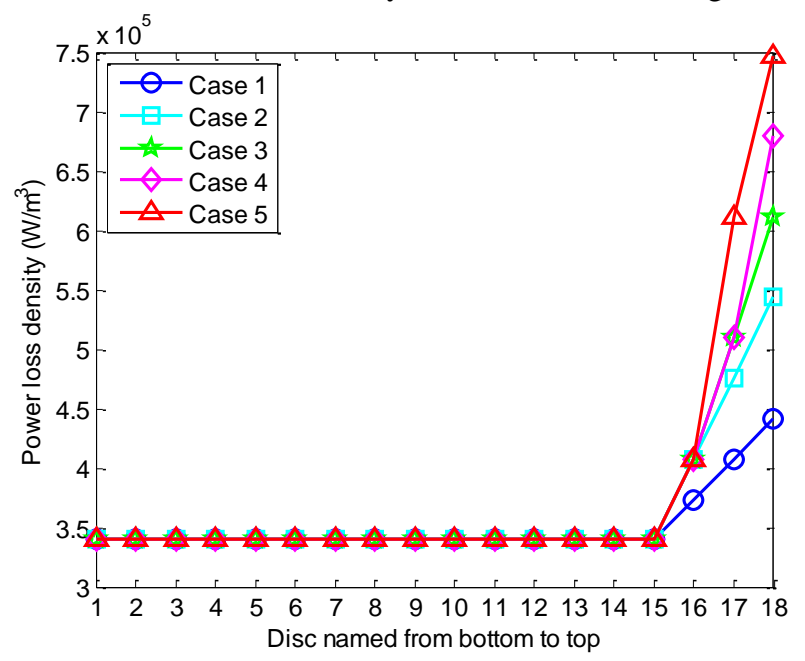

Fig. 6. Nonunifrom loss distribution in the winding model.
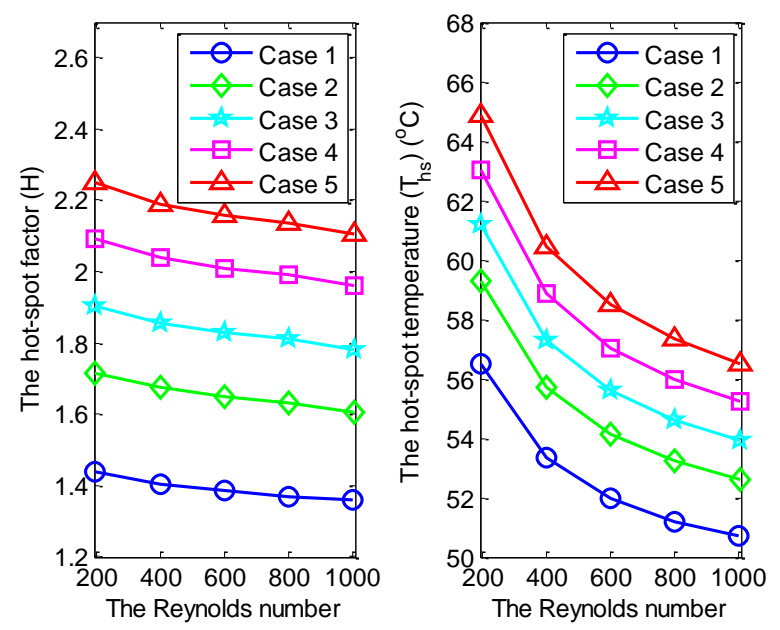

Fig. 7. The variations of $H$ and $T_{h s}$ with Re for nonuniform-loss cases.

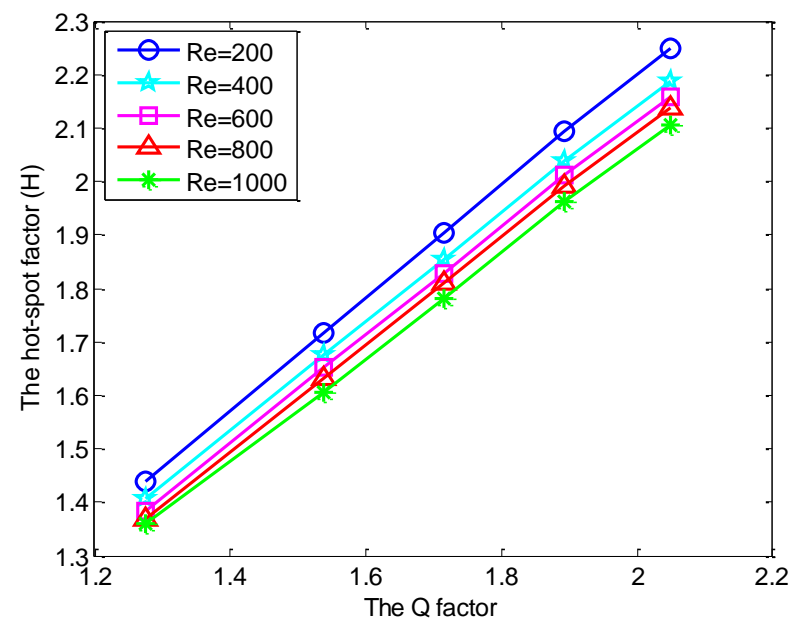

Fig. 8. The variation of $H$ with the $Q$ factor for different Reynolds numbers. 


\section{DISCUSSIONS}

The hot-spot factor, $H$, is a crucial component of the thermal diagram for determining the hot-spot temperature from temperature rise test data. The linearity assumptions in the thermal diagram, however, make the concept of $H$ more empirical than theoretical. Therefore, $H$ is still open to better interpretation.

\section{A. Interpretation of the Hot-Spot Factor}

This paper presents a different interpretation of $H$ based on dimensional analyses of the fluid flow and heat transfer processes in the winding, rather than the linearity assumptions in the thermal diagram.

According to the dimensional analysis shown in section II part $C, H$ is interpreted as the dimensionless temperature at the hot-spot. The locations of the lumped temperatures $\left(\mathrm{T}_{\mathrm{aw}}, \mathrm{T}_{\mathrm{to}}\right.$, $\mathrm{T}_{\mathrm{bo}}$ ) in the definition of $H$ do not affect the physical meaning of $H$, but will affect the magnitude of $H$ derived. For example, wherever the location of the top oil temperature is defined, top of the winding or top of the tank, once the location is fixed the top oil temperature will no long be a function of the coordinates and therefore the same dimensionless governing equations will be applicable, giving $H$ the same physical meaning.

\section{B. On the Experimentally Derived $H$ from CIGRE Report}

A statistic of $H$ based on optical fibre measurements during heat run tests was previously provided in [9] when CIGRE conducted work on experimental determination of power transformer hot-spot factor. For OD transformers, 27 hot spot factors were obtained with the minimum being 0.51 and the maximum 1.83; statistically resulting in a mean of 1.18 with standard deviation of 0.29 .

The hot spot factors less than 1 are likely due to the fact, apart from measurement errors, that the installation of fibre-optic temperature sensors in the radial spacers unintentionally allowed oil flowing into the sensor area which resulted in the temperature in the fluid domain being measured. Therefore, the hot-spot factors derived might be their convective components, $\mathrm{H}_{\mathrm{ve}}$, which was introduced previously in this paper and can be less than 1 due to the presence of the paper insulation in the winding. As an example, when Re is less than 1000 , the convective hot-spot factors obtained for the uniform loss cases in section III part B are always less than 1 . Another likely reason could be that the temperature measured was not the hot-spot temperature because the hot-spot can be located at the bottom of the top pass, as shown in Fig. 2 (b).

The maximum hot spot factor was 1.83 for the measured OD transformers in the CIGRE report, such a scenario could occur as the case demonstrated when Re is as high as 1200 for the winding studied shown in Fig. 4. The mean of the hot spot factors obtained being 1.18 in the report is close to the hot spot factors in Fig. 4 when $\mathrm{Re}$ is around 600, which should be regarded as an optimal operational regime.

It is worth emphasizing that Fig. 4 and the correlation coefficients in Table III for (21) are specific to the winding investigated under uniform loss distribution condition. However, the general trend of $H$ variation with Re and $\operatorname{Pr}$ and the magnitude of $H$ shown in Fig. 4 would be representative for all winding geometries. The reasoning is as follows: oil flow distribution in the winding tends to be reasonably uniform when $\mathrm{Re}$ is moderate corresponding to a moderate total oil flow rate and moderate oil temperature [14], which would lead to a reasonably uniform temperature distribution and a hot spot factor close to 1 . On the other hand, with the increase of Re, which is usually due to an increase of total oil flow rate, flow distribution would start to get distorted [14]. Hence the effect of increasingly distorted flow distribution competes with the effect of increased total oil flow rate, and results in a slightly fluctuating or stable hot spot factor. However, when Re is increased too much, the flow distribution becomes severely distorted and reverse flow even occurs [14], and this can jeopardize the cooling performance, so high hot spot factors are observed. For OD cooling modes, it is not necessarily the higher the total oil flow rate the better the cooling performance. In a word, the influence of operational conditions, which can affect $\operatorname{Re}$ and $\operatorname{Pr}$, has been investigated in this paper for a given winding geometry. If the influence of geometrical parameters is to be taken into account for (21), CFD geometrical parameter sweeps are needed, of which the method is shown in [14].

\section{Influences of Operational Conditions on $H$}

Heat transfer analysis decomposes $H$ into two separable components: $H_{d u}$ and $H_{v e}$. Both components are related to dimensionless winding geometry, power loss distribution, Re and Pr. With this decomposition and the understanding of the two components, $H$ for OD transformers can be better understood in terms of how it is controlled by the ambient temperature, the total oil flow rate, and the loading level.

\section{1) Influence of ambient temperature}

The ambient temperature affects $H$ indirectly through changing oil properties to change Re and Pr. Therefore, the effect of ambient temperature on $H$ depends on both the fluctuation range of ambient temperature, oil property sensitivity to temperature and transformer operational regime. One of the cases in section III-B-2 can be taken as an example to demonstrate the influence of ambient temperature on $H$. The chosen case was shown in Fig. 5, where the winding inlet velocity is $0.4 \mathrm{~m} / \mathrm{s}$; the power loss density is $3.397 \times 10^{5} \mathrm{~W} / \mathrm{m}^{3}$ (equivalent to a current density of $4 \mathrm{~A} / \mathrm{mm}^{2}$ ); the average oil temperature is $40^{\circ} \mathrm{C}$; Re and $\operatorname{Pr}$ are 700 and 107 , respectively. To demonstrate the influence of ambient temperature on $H$, two reasonable assumptions are further made. First, the average oil temperature of $40^{\circ} \mathrm{C}$ corresponds to an ambient temperature of $20{ }^{\circ} \mathrm{C}$, i.e. average oil temperature rise over ambient is a constant of $20 \mathrm{~K}$ for the investigated winding model. Second, when the ambient temperature changes the total oil flow rate and power losses in the winding remain unchanged. Therefore, the average oil temperature changes linearly with the ambient temperature, subsequently changing $\operatorname{Re}$ and Pr. The variation of $H$ with ambient temperature for the case can then be obtained through (21) as shown in Fig. 9.

As can be seen in Fig. 9, the variation of ambient temperature can have either a negligible or profound influence on $H$. On one hand, $H$ does not change significantly when the ambient temperature is lower than $20^{\circ} \mathrm{C}$ (this is corresponding to a regime when the transformer is operated with $\operatorname{Re}<700$ shown in Fig. 4), so variation of ambient temperature when lower than 20 ${ }^{\circ} \mathrm{C}$ has a negligible influence on $H$. However, on the other hand when the ambient temperature is higher than $20^{\circ} \mathrm{C}$ the increase 
of ambient temperature can result in a profound influence on $H$, as this corresponds to a critical regime of Re>700 shown in Fig. 4 , where a moderate increase of $\mathrm{Re}$ can result in an obvious increase of $H$.

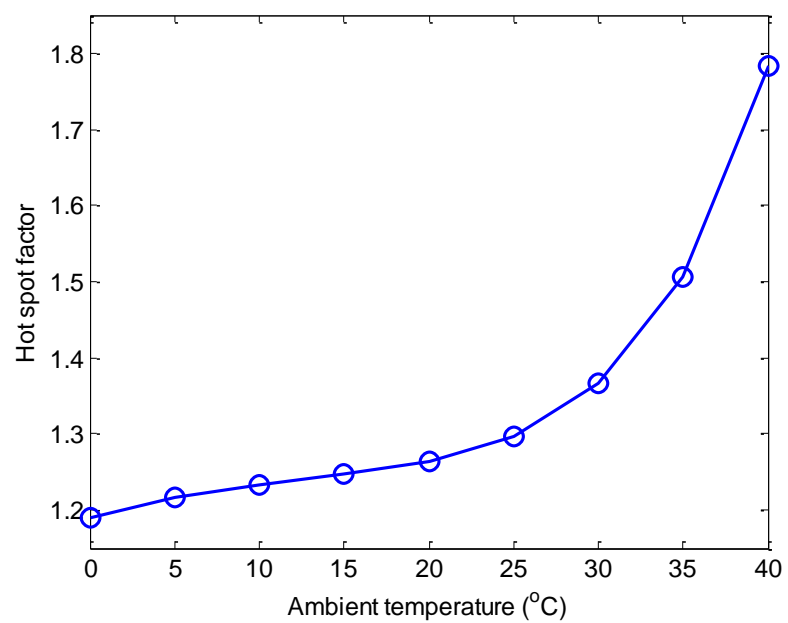

Fig. 9.Example of influence of ambient temperature on hot spot factor.

\section{2) Influence of total oil flow rate}

The total oil flow rate determines Re directly. Depending on flow distribution and loss distribution, the total oil flow rate can have a moderate influence on $H$ if it is a uniform loss distribution with $\operatorname{Re}<600$, as shown in Fig. 4, or have a significant influence if it is a uniform loss distribution with $\mathrm{Re}>600$, or a distorted power loss distribution, as shown in Fig. 7 (a).

\section{3) Influence of loading level}

The influence of the loading level or the power loss distribution on $H$ is from the $Q^{\prime}$ instead of the magnitude of the loss, i.e. $H$ is not related to load factor, $\mathrm{K}$, but $\mathrm{Q}^{\prime}$, as shown in Fig. 8.

In general, the hot-spot locates at the point of the highest power loss as shown in section III part $\mathrm{C}$-unless the flow is so distorted that a serious local overheating is resulted, which anyway should be avoided. Therefore, $Q^{\prime}$ can be regarded to be the same as $Q$ and by so doing the hot-spot temperature could be slightly over estimated.

For a fixed winding geometry, when the hot-spot is at the point of the highest power loss, $H$ is in a linear relationship with $Q$ and this linear relationship is coupled with the nonlinear relationship between $H$ and Re and Pr. The Prandtl number is much less influential than Re. Therefore, according to (16) and the dimensional analyses on fluid flow and heat transfer, $H$ can be expressed qualitatively as:

$$
H=f_{s}(\operatorname{Re}, \operatorname{Pr}) \cdot Q+f_{i}(\operatorname{Re}, \operatorname{Pr}) \approx f_{s}{ }^{\prime}(\operatorname{Re}) \cdot Q+f_{i}{ }^{\prime}(\operatorname{Re})
$$

where $f_{s}(\operatorname{Re}, \operatorname{Pr})$ and $f_{s}^{\prime}(\operatorname{Re})$ represent the slope term $h^{\prime} / h$ $+h^{\prime} \cdot d x / k_{p}$ and $f_{i}(\mathrm{Re}, \mathrm{Pr})$ and $f_{i}^{\prime}(\mathrm{Re})$ represent the intercept term $g^{\prime} / g$.

For the investigated cases in section III part C, when $\mathrm{Re}$ ranges from 200 to 1000 , the slope term ranges from 0.969 to 1.05 and the intercept term ranges from 0.100 to 0.122 . The slope term and the intercept term are quite constant, indicating the importance of having a moderate $Q$ factor because $Q$ is almost directly added into $H$.

Compared to the formula in IEC 60076-2: 2011, $H=S \times Q$, (22) replaces the $S$ factor with $\operatorname{Re}$ and $\operatorname{Pr}$ (or solely with $\mathrm{Re}$ ) and changes the formula as well.

\section{On Winding Temperature Indicator}

The quantitative relationship between $H$ and the dimensionless controlling parameters, $Q, \operatorname{Re}$ and $\operatorname{Pr}$ for a transformer can be obtained through experimental or numerical parametric sweeps. When the relationship is quantified, the real-time hot-spot factor can be determined according to real-time operational conditions and therefore the real-time hot-spot temperature can be obtained through a winding temperature indicator, which is widely used to control the operation of the pumps and fans.

\section{CONCLUSION}

The hot-spot factor, $H$, is interpreted as the dimensionless temperature at the hot-spot based on dimensional analyses of fluid flow and heat transfer. $H$ is decomposed into the sum of two separable components: the conductive component, $H_{d u}$, and the convective component, $H_{v e}$. For a fixed winding geometry with a fixed power loss distribution in dimensionless senses in OD cooling modes, no matter it is uniform or not, both components are functions of $\mathrm{Re}$ and $\mathrm{Pr}$.

The relationship between $H$ and Re and Pr for uniform-loss cases has been obtained by performing CFD parametric sweeps and correlating the hot-spot factors from CFD results with Re and Pr. The correlation has been verified by the consistency between the hot-spot factors obtained from new CFD simulations and the corresponding ones obtained from the correlation.

When the loss distribution is a variable, $H$ is a function of the $Q$ factor, Re and Pr. For the condition of the hot-spot at the location of the highest power loss, $H$ is in a linear relationship with $Q$ and this linear relationship is coupled with the nonlinear relationship between $H$ and $\operatorname{Re}$ and Pr. Therefore, the overall relationship between $H$ and $\mathrm{Q}, \operatorname{Re}$ and $\operatorname{Pr}$ is nonlinear and experimental or numerical parametric sweeps are needed to quantify the relationship.

\section{ACKNOWLEDGMENT}

The authors would like to express their gratitude to M\&I Materials, National Grid, Scottish Power, Shell, TJ|H2b Analytical Services, UK Power Networks and Weidmann Electrical Technology for their financial and technical contributions to the Transformer Research Consortium at The University of Manchester.

\section{REFERENCES}

Loading guide for oil-immersed power transformers, IEC standard IEC 60076-7, 2005.

IEEE Guide for Loading Mineral-Oil-Immersed Transformers and Step-Voltage Regulators, IEEE standard C57.91, 2011.

W. Lampe, L. Pettersson, C. Ovren et al., "Hot-spot measurements in power transformers," CIGRE, Pep. 12-20, 1984.

H. Nordman, and M. Lahtinen, "Thermal overload tests on a 400-MVA power transformer with a special 2.5-pu short time loading capability," IEEE Trans. Power Del., vol. 18, no. 1, pp. 107-112, 2003. 
[5] P. Picher, F. Torriano, M. Chaaban et al., "Optimization of transformer overload using advanced thermal modelling." CIGRE Pep. A2-305, 2010.

[6] A. J. Oliver, "Estimation of transformer winding temperatures and coolant flows using a general network method," Proc. Inst. Elect. Eng., vol. 127, pp. 395-405, 1980.

[7] Temperature rise for liquid-immersed transformers, IEC standard 60076-2, 2011.

[8] Z. Radakovic, U. Radoman, and P. Kostic, "Decomposition of the Hot-Spot Factor," IEEE Trans. Power del., vol. 30, pp. 403-411, 2015.

[9] "Experimental determination of power transformer hot-spot factor," CIGRE WG 12-09, Electra no. 161, August 1995.

[10] D. Feng, Z. Wang, and P. Jarman, "Evaluation of Power Transformers' Effective Hot-Spot Factors by Thermal Modeling of Scrapped Units," IEEE Trans. Power Del., vol. 29, no. 5, pp. 2077-2085, 2014.

[11] F. Torriano, P. Picher, and M. Chaaban, "Numerical investigation of 3D flow and thermal effects in a disc-type transformer winding," Appl. Therm. Eng., vol. 40, pp. 121-131, 2012.

[12] X. Zhang, Z. D. Wang, Q. Liu, A. Gyore, P. Jarman, P. Dyer, "Investigation of Convective Heat Transfer Efficiency in the Horizontal Cooling Duct of a Disc Type Transformer Winding," Paper ID 280, 19th ISH, Czech Republic, 2015.

[13] T. L. Bergman, F. P. Incropera, and A. S. Lavine, Fundamentals of heat and mass transfer: John Wiley \& Sons, 2011.

[14] X. Zhang, Z. Wang, and Q. Liu, "Prediction of Pressure drop and flow distribution in disc type transformer windings in an OD cooling mode," IEEE Trans. Power Del., 2016, DOI (identifier) 10.1109/TPWRD.2016.2557490, In press.

[15] M. Heathcote, $J \& P$ transformer book, Thirteenth ed.: Newnes, 2007.

[16] F. Torriano, M. Chaaban, and P. Picher, "Numerical study of parameters affecting the temperature distribution in a disc-type transformer winding," App. Therm. Eng., vol. 30, no. 14, pp. 2034-2044, 2010.

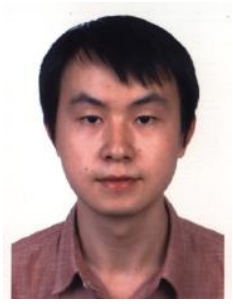

Xiang Zhang received the B.Eng. degree in Electrical and Electronic Engineering from Xi'an Jiaotong University, Xi'an, China, in 2012 and is currently pursuing the $\mathrm{Ph}$.D. degree at power and energy division of the School of Electrical and Electronic Engineering at The University of Manchester, Manchester, UK. His research programme is on transformer thermal modelling.

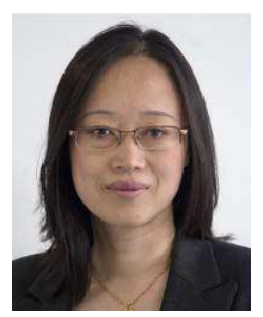

Zhongdong Wang received the BSc and the MSc degrees in high voltage engineering from Tsinghua University in 1991 and 1993 and the $\mathrm{PhD}$ degree in electrical engineering from UMIST in 1999. Currently she is a Professor of High Voltage Engineering of the School of Electrical and Electronic Engineering at The University of Manchester. Her research interests focus on power transformers covering all aspects of modelling and simulation, materials \& systems, and asset management policies.

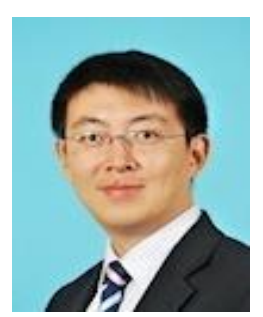

Qiang Liu (S'08-M'12) obtained the B.Eng. degree in electrical engineering (2005) and the M.Eng. degree in high voltage and electrical insulation (2008) from Xi'an Jiaotong University in China, and the Ph.D. degree in electrical power engineering (2011) from The University of Manchester in the UK. Currently he is a Senior Lecturer of the School of Electrical and Electronic Engineering at The University of Manchester. His research interests are on pre-breakdown and breakdown phenomena in liquids, ester transformer liquids, streaming electrification, ageing of insulating materials, transformer asset management and high voltage testing. 